\title{
Whole exome sequencing of patients who resolved Crohn's disease and complex regional pain syndrome following treatment for paratuberculosis
}

\author{
J. Todd Kuenstner ${ }^{1 *}$, Maher Kali ${ }^{2}$ and Christine Welch ${ }^{3}$
}

\begin{abstract}
Background: A whole exome sequencing study was performed on an extended family including a patient with Crohn's disease (CD) and a patient with complex regional pain syndrome (CRPS). The patient with CD and the patient with CRPS have experienced resolution of their disease following treatment for paratuberculosis. The study was performed in order to determine if there is an unusual mutation in this extended family that would explain the susceptibility to mycobacterial infection among many of the members.
\end{abstract}

Results: We identified sets of rare single nucleotide polymorphisms (SNPs) that were shared among affected family members, including variants in two genes, IL15RA and CASP10, which have established roles in the immune response. In addition, the CD and CRPS patients were found to have heterozygous mutations in MBL2 and DDX58, mutations that have been associated with susceptibility to tuberculosis.

Conclusions: The IL15RA and CASP10 variants may contribute to the disease symptoms exhibited in this family. The finding of SNPs associated with immune function supports a complementary role of infection and genetics in these diseases.

Keywords: Mycobacterium avium ssp. paratuberculosis, Crohn's disease, Complex regional pain syndrome, Lymphangiomatosis, Type 1 diabetes mellitus, Genetic susceptibility, Single nucleotide polymorphism

\section{Background and aims}

Most research in Crohn's disease (CD) has focused on genetics rather than the association of the disease with infection by Mycobacterium avium ssp. paratuberculosis (MAP) [1-4]. Over 140 genetic mutations have now been identified in CD [5]. These two areas of research are probably complementary because some of the genetic mutations, may indicate increased susceptibility to MAP infection. For example, the NOD2 mutation found in some CD patients is associated with susceptibility to MAP infection in cattle [6]. Furthermore, a 2009 study from China showed that patients with leprosy

\footnotetext{
${ }^{*}$ Correspondence: jtodd.kuenstner@tuhs.temple.edu

1 Department of Pathology, Temple University Hospital, 3401 N. Broad St. Philadelphia, PA 19140, USA

Full list of author information is available at the end of the article
}

and patients with CD have higher frequencies of NOD2, TNFSF15 and IL12B mutations than healthy controls [7, 8]. A large meta-analysis genome-wide association study (GWAS) concluded that there is considerable overlap between the susceptibility loci for inflammatory bowel disease (IBD) and mycobacterial infection [9].

In a recent case series report, four patients with multiple diseases of unknown etiology (cases 1-4) and one healthy individual (case 5) were found to have evidence of infection by MAP including positive blood cultures (except in case 4 which had positive MAP antibodies). Two of the cases (case 1 with $C D$ and asthma and case 2 with CRPS, hypothyroidism and Raynaud's phenomenon) were treated with a combination of anti-MAP antibiotics and ultraviolet blood irradiation (UVBI) with resolution of the diseases and inability to culture MAP in post 
treatment blood samples. These case reports of patients with MAP infections provide supportive evidence of a pathogenic role of MAP in humans [10].

The role of genetic mutations which might confer increased susceptibility to mycobacterial infection and immune disorders was investigated by whole exome analysis in this family with an extensive history of mycobacterial infection and diseases traditionally considered autoimmune (see Fig. 1).

\section{Results}

We recruited members of a single family where individuals were affected by asthma, CD, hypothyroidism and CRPS and performed whole exome sequencing on 10 members of the family (see Fig. 1 and Table 1).

We searched for variants present in any of five CD susceptibility genes (ATG16L1, IL23R, CARD9, NOD2, and PTGER4) in any affected family member using models 1 , 2 and 5 in Table 2 . We detected no variants that met the criteria in any affected family member under any of the three models. Removal of the requirement for absence in the normal family member revealed two variants which were found under all three models: (1) ATG16L1 rs2241880, a missense variant (Thr318Ala), has an allele frequency of 0.456 and is rated as benign by Polyphen2 and tolerated by SIFT and (2) IL23R rs7530511 a missense variant (Leu310Gln) has a frequency of 0.166 and is also rated as benign.

We also searched our variant data sets for established tuberculosis susceptibility genes and loci that had variants present in any affected family member using three autosomal dominant models. In this analysis, we used the filter chains for models 1,2 and 5 in Table 2 . with the following modifications: (1) The minor allele frequency and Polyphen filters were removed, (2) Sequence ontology was expanded to include $5^{\prime}$ and $3^{\prime}$ UTRs, and (3) A filter that selected for variants present in DDX58, IL10, IL12B, MBL2, SLC11A1 and two Tuberculin Skin Test Reactivity loci (TST1 11p14-15, TST2, 5p15) was added [11-14]. Under the modified versions of models 1,2 and 5 , we identified 12,13 and 10 variants, respectively. Gene names, variant ID numbers, predicted pathogenicity and variant allele frequencies are provided in Additional file 1: Appendix 1.

Next, we investigated whether a genetic variant with pleiotropic effects could cause clinically different presentations in affected family members and whether a single variant might underlie these presentations. In order to identify variants that could contribute to the disease state, we performed discrete filtration under three autosomal dominant (AD) models and three autosomal recessive (AR) models found in Table 2. In models 1 (AD) and 3 (AR) we searched for variants that were shared by those who were affected by asthma (patient 004), CRPS (patient 005), CD (patient 006) and diabetes (patient 012) and absent from a paternal normal (patient 003). In models $2(\mathrm{AD})$ and $4(\mathrm{AR})$, we required that the disease variant was present in patients with asthma (patient 006), CD (patient 006), CRPS (patient 005), or hypothyroidism (patients 005, 008, 009, 011 and 014) and absent in a normal maternal relative (patient 010 ). We also sought shared variants under models based on mycobacterial infection of family members 004, 005, 006 and 012 under an autosomal dominant model (\#5) and an autosomal recessive model (\#6).

We constructed discrete filtration chains using VarSeq software in order to identify shared genetic variants (single nucleotide variants (SNVs) and small indels); filter sets for each chain are shown in Table 2. The number of variants retained after each step in the filtration chain is shown in Table 3. Under models 1, 2, 3, 4, 5, 6, we identified $57,56,1,14,41$ and 1 variants, respectively, that were shared in all affected members. Since the disease state of some affected members may stem from locus heterogeneity (i.e. different genetic etiologies), we repeated the analysis with the six additional filtration chains (models 7-12 in Table 2) where we required $\mathrm{N}-1$ members of the affected group to share a variant instead of all affected members. As expected, reduction of stringency in the shared variant step increased the numbers of variants for most of the models (Table 3).

In order to assess the significance of the shared variants from models 1-12, we collapsed variants into unique genes (gene symbols), entered each symbol into the GeneCards and MalaCards databases $[15,16]$ and generated lists of shared genes and associated disease states (Additional file 1: Appendix 2). We then searched these lists for the term autoimmune or interleukin. Two variants were identified in these searches: (1) a variant in Interleukin 15 receptor alpha (IL15RA, rs8177777) is shared by all affected members under models 1, 5, 7 and 11 . The allele frequency of rs8177777 is 0.01 in the ExAC database [17] and 0.0082 in the 1000 Genomes database [18]. All affected members are G/A heterozygotes. IL15RA encodes the alpha subunit of a cytokine receptor which binds interleukin 15 (IL15) and is critical to the early immune response to microbial infections. The variant causes a missense substitution of alanine to valine at position 97 (Ala97Val) in IL15RA isoform X1. MutationTaster, a predictor of missense functional consequences rated this variant as tolerated [19] (2) Using models 2 and 8 , all affected members were heterozygous for SNP rs17860405 which leads to a tyrosine to cysteine missense at position 446 (Tyr446Cys) in the caspase 10 protease (CASP10). Its allele frequency is 0.0295 in the ExAC database and 0.0128 in the 1000 Genomes database. 


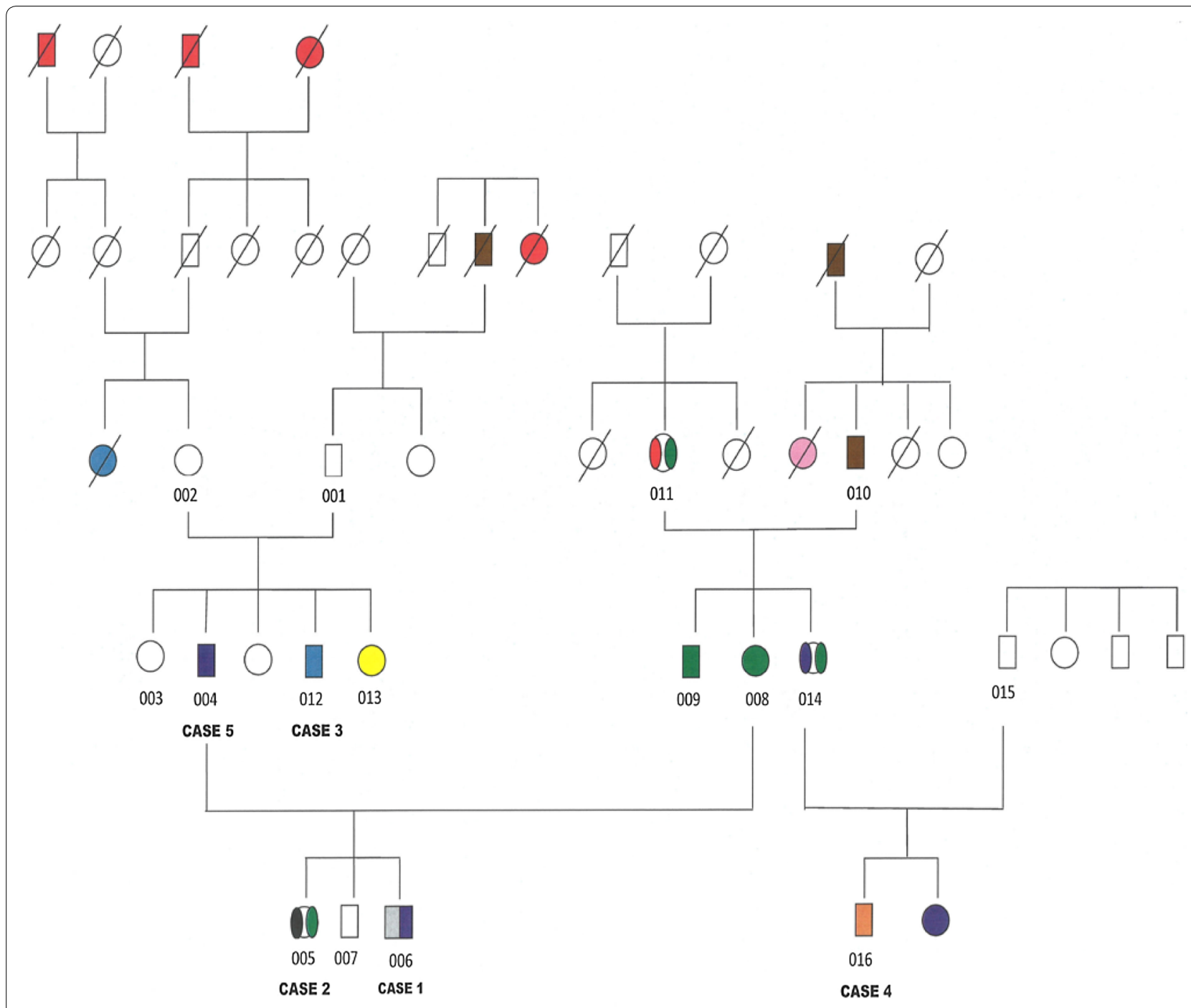

Legend:

Red Tuberculosis

Blue Diabetes Mellitus Type 1

Green Hypothyroidism

Yellow Hyperthyroidism

Purple Asthma

Gray Crohn Disease

Orange Lymphangiomatosis

Black Complex Regional Pain Syndrome

Pink Rheumatoid Arthritis

Brown Chronic Lymphocytic Leukemia

Fig. 1 Family pedigree summarizing the history of mycobacterial infection and other diseases in cases 1 through 5 and in additional family members. Each study subject number (001-016) appears beneath the corresponding subject. Cases 1-5 are 006, 005, 012, 016 and 004, respectively. This figure previously appeared in reference 10 and is used with permission of the publisher 
Table 1 Whole exome read and alignment data

\begin{tabular}{|c|c|c|c|c|c|}
\hline Sample ID & Total reads pass filter & Total aligned reads & $\begin{array}{l}\text { Percent aligned } \\
\text { reads }\end{array}$ & $\begin{array}{l}\text { SNP percent found } \\
\text { in dbSNP }\end{array}$ & Mean coverage \\
\hline 315-001-003 & $85,396,980$ & $78,226,223$ & 91.6 & 98.8 & $41.1 X$ \\
\hline 315-001-004 & $116,661,046$ & $114,816,183$ & 98.4 & 98.8 & $58.9 x$ \\
\hline 315-001-005 & $111,609,738$ & $109,886,187$ & 98.5 & 98.9 & $57.3 x$ \\
\hline 315-001-006 & $140,466,506$ & $137,765,626$ & 98.1 & 98.9 & $72.8 X$ \\
\hline 315-001-008 & $133,788,894$ & $126,633,454$ & 94.7 & 99.2 & $65.8 \mathrm{X}$ \\
\hline 315-001-009 & $119,135,414$ & $115,697,404$ & 97.1 & 98.8 & $57.6 X$ \\
\hline $315-001-010$ & $114,281,892$ & $111,467,858$ & 97.5 & 99 & $57.2 X$ \\
\hline 315-001-011 & $133,211,626$ & $130,104,251$ & 97.7 & 98.7 & $72.9 x$ \\
\hline $315-001-012$ & $139,647,612$ & $136,534,857$ & 97.8 & 98.8 & $78 x$ \\
\hline 315-001-014 & $170,815,864$ & $168,254,316$ & 98.5 & 98.9 & $99.5 X$ \\
\hline
\end{tabular}

Polyphen and SIFT rate this variant as possibly damaging [20] and tolerated [21], respectively.

Finally, we searched for whole exome variant sets for variants in ORMDL3 (mutations observed in CD and asthma) under four models: autosomal dominant with affected parental members (model \#1), autosomal recessive with affected parental members (model \#2), autosomal dominant with mycobacterial susceptible members (model \#5), and autosomal recessive with mycobacterial susceptible members (model \#6). All four of these models invoked 4, 5, 6 and 12 as affected subjects.

In each case, the filtration chain was modified to resemble previous searches for named MS genes that we previously did. These changes included (a) expanding the sequencing ontology to include $5^{\prime}$ and $3^{\prime}$ UTRs and synonymous missense variants, (b) removing the allele frequency filter (so that alleles with any frequency would be allowed), (c) removing the requirement for sharing variants among the four affected members $(4,5,6$ and 12) such that the presence of a variant in any of the affected members would be allowed, and (d) removing the Polyphen pathogenicity filter. The filter which required a read depth of 10 or more for each variant call was retained. The sequence ontology filter excluded intronic and intergenic variants as did the named gene searches. In spite of the expanded filtration design, no ORMDL3 variants in subjects 4, 5, 6 and 12 were found under any of the models.

\section{Discussion}

In this study, none of the affected patients were found to have any of 5 common mutations in CD. The CD and CRPS patients were found to have heterozygous mutations in MBL2 and DDX58, mutations that have been associated with susceptibility to tuberculosis.

In addition, we identified several rare genetic variants which were shared among affected members under twelve genetic models. A few variants were located in genes with known roles in the immune response (CASP10 and IL15RA).

For the cases in this study, the heterozygous combination of mutations of MBL2 and DDX58 may indicate increased susceptibility to mycobacterial infection. However, this and the previous study of this extended family suggests that the more important factor in the diseases of these hosts was MAP bacteremia and the presence and absence of MAP corresponded to the presence and resolution of several diseases. Moreover, a much greater percentage of patients with $\mathrm{CD}$ have evidence of MAP infection than those with any single SNP identified in this cohort of patients or any other cohort of CD patients also suggesting that MAP infection plays a more constant and important role in $\mathrm{CD}$ than the genetic constitution of the affected patients.

\section{Conclusions}

The ever expanding number of genetic mutations associated with CD (over 140 susceptibility loci in 2014) [5], the increasing incidence and prevalence of $C D$ [22], the corresponding increasing prevalence of MAP infection in cattle [23], and the recently published case series report with supportive evidence of the pathogenicity of MAP in humans [10] implicate MAP infection as an important candidate trigger of $\mathrm{CD}$. Controlled clinical trials using a combination of UVBI and antibiotics to determine whether clearance of MAP bacteremia results in CD resolution in other patients are indicated.

\section{Methods}

Patient recruitment and confidentiality: 16 family members of an extended family with multiple diseases of unknown etiology were recruited for the study following review and approval by the Charleston Area Medical Center Institutional Review Board (Institutional 

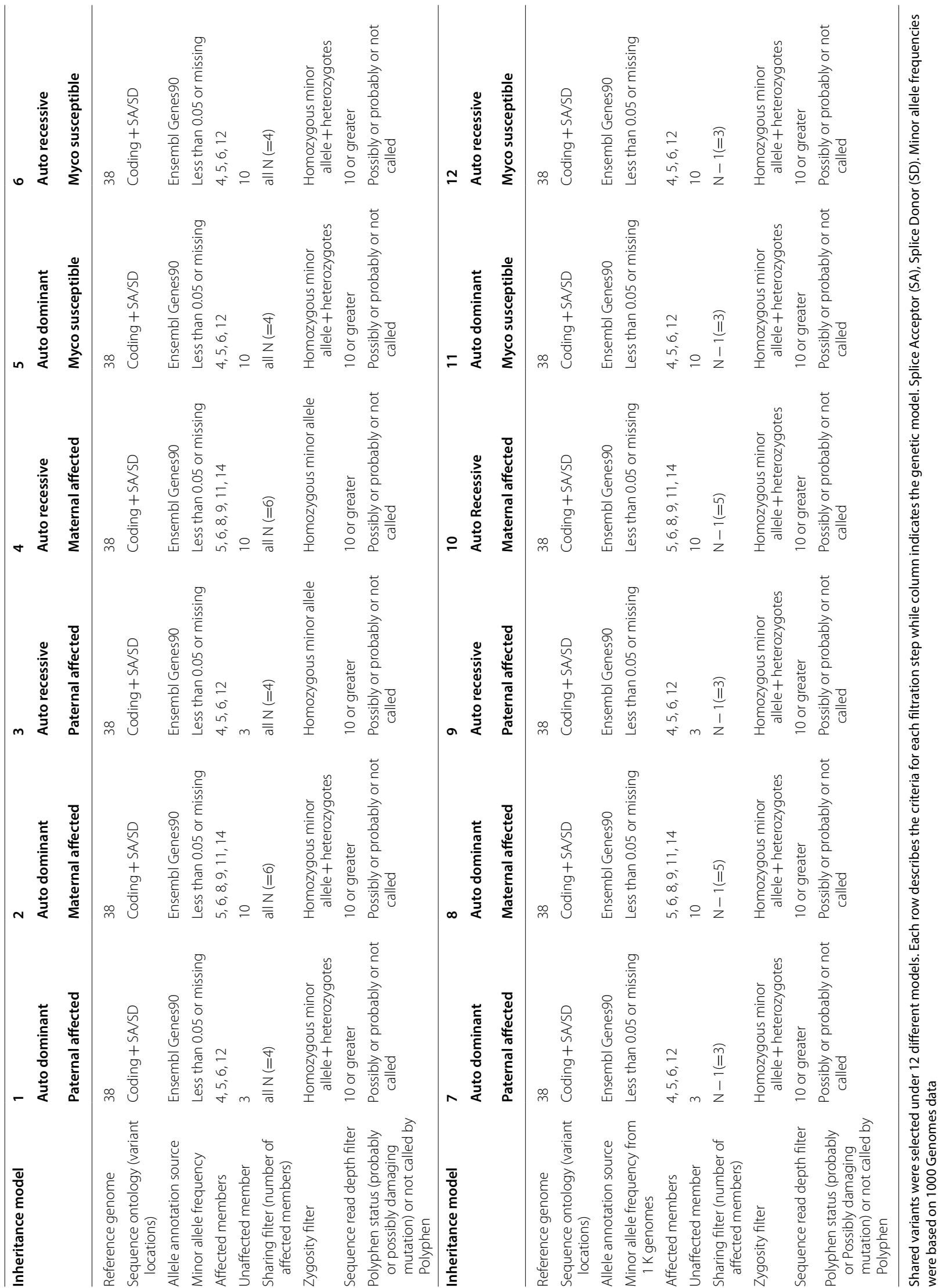
Table 3 Discrete filtration

\begin{tabular}{|c|c|c|c|c|c|c|}
\hline Inheritance model & 1 & 2 & 3 & 4 & 5 & 6 \\
\hline Sequence ontology & 24,623 & 26,950 & 24,623 & 26,950 & 29,197 & 29,612 \\
\hline European allele frequency $\leq 0.05$ or missing & 11,024 & 12,864 & 3144 & 13,899 & 15,089 & 15,592 \\
\hline Variants shared among affected members & 903 & 655 & 974 & 1432 & 1869 & 1500 \\
\hline Variants absent from normal or not called & 110 & 96 & 2 & 16 & 73 & 2 \\
\hline Sequence read depth $\geq 10$ for all patients & 95 & 89 & 1 & 15 & 68 & 1 \\
\hline Polyphen = possibly or probably damaging or & 57 & 56 & 1 & 14 & 41 & 1 \\
\hline Inheritance model & 7 & 8 & 9 & 10 & 11 & 12 \\
\hline Sequence ontology & 24,623 & 26,950 & 24,623 & 26,949 & 29,197 & 29,612 \\
\hline European Allele $\leq$ frequency 0.05 or missing & 11,024 & 12,864 & 3144 & 13,898 & 15,089 & 15,592 \\
\hline Variants shared among affected members & 2228 & 1213 & 1012 & 1636 & 3239 & 1816 \\
\hline Variants absent from normal or not called & 577 & 292 & 6 & 34 & 353 & 14 \\
\hline Sequence read depth $\geq 10$ for all patients & 142 & 111 & 2 & 17 & 73 & 3 \\
\hline Polyphen $=$ possibly or probably damaging or & 91 & 74 & 1 & 16 & 45 & 2 \\
\hline
\end{tabular}

We screened for relevant variants using twelve different genetic models as described in Table 2. Rows provide the number of variants following application of each filtering criterion

Review Board \#1,997,444, April 29, 2014). Recruitment was conducted by the Charleston Area Medical Center Clinical Research Institute. Subjects were assigned a continuously-running study identification number to maintain confidentiality of records. Only de-identified data was shared with genomic data analysts. Subject data records were kept in a locked filing cabinet. Electronic subject data was maintained on password protected computers. All subject data were kept in strict confidence.

Genomic DNA isolation and whole exome sequencing: Buccal samples were collected from 15 of the 16 consented family members (subject 016 (case 4) did not submit a buccal swab) using a protocol designed for genomic DNA isolation. Each participant was asked not to consume any food or drink in the $30 \mathrm{~min}$ prior to sample collection and collected their own swab by placing the swab inside their mouth and scraping it firmly against the inside of each cheek 6 times. Buccal samples were then air-dried for at least $2 \mathrm{~h}$ after collection. After drying, the swab head was ejected into a sterile $2 \mathrm{ml}$ screw cap microfuge tube which was labeled with patient study number. These samples were then shipped overnight in batches at room temperature to the Marshall University Genomics Core Facility (Huntington, WV) where the genomic analysis was performed.

Genomic DNA was extracted from buccal samples from fifteen patients using a QiaAmp DNA Mini kit (Qiagen, Germantown, MD) using the method described by the manufacturer and quantified Qubit fluorimetry (ThermoFisher Scientific, Waltham, MA). A $260 / 280$ absorbance ratios were in the 1.70 to 2.00 range for all samples. Indexed whole exome libraries were prepared from $50 \mathrm{ng}$ of genomic DNA using Nextera Whole Exome enrichment kits (Illumina Inc., San Diego, California). Library yields were quantified by Qubit fluorimetry and insert sizes were determined by Agilent Bioanalyzer electrophoretic analysis. Insert sizes ranged from 273 bp to $700 \mathrm{bp}$. The resulting fifteen whole exome libraries were clustered on an Illumina cBot cluster station and sequenced in a $2 \times 50$ paired end strategy on an Illumina HiSeq 1500 running in the high output mode. Whole exome sequence data was analyzed using the BWA Enrichment application on Illumina Base Space. For the 10 libraries used in the filtration analysis described below, total reads/library ranged from 85,396,980 to $170,815,864$. Percent aligned reads ranged from 91.6 to 98.8\%. Alignments identified between 99.1 and $99.3 \%$ of SNPs found in dbSNP and between 90.9 and $93.6 \%$ of indels found in dbSNP. The mean coverage depth ranged from $41.1 \mathrm{X}$ to $107.1 \mathrm{X}$. A summary of the whole exome read and alignment data appears in Table 1.

Reads were aligned to GRCh38 using Bowtie v2.0.1 [24]. Small variants (SNPs and indels) were identified using Samtools v0.1.18 [25] and output as VCF files. We filtered for potential causal variants under six different Mendelian models using VarSeq software v1.5.0 (Golden Helix, Bozeman MT). In general, VarSeq filtration chains were designed to identify low frequency variants that encoded pathogenic changes shared by all affected family members under the specified Mendelian model. Each chain contained the following filters: (1) A sequence ontology filter classified and collected variants that were frameshift, missense, splice acceptor, splice donor, splice region variant, initiator codon, stop gain, stop loss, stop-retained mutations, or inframe deletions or insertions. We excluded variants 
that led to synonymous codon changes and variants that were located in intergenic, intronic and $5^{\prime}$ and $3^{\prime}$ UTR regions. (2) An allele frequency filter retained variants whose frequency was $\leq 5 \%$. (3) A zygosity filter retained heterozygotes or variant homozygotes in the autosomal dominant mode or variant homozygotes in the autosomal recessive mode. (4) A shared variant filter retained variants that were common to all of the affected members or an $\mathrm{N}-1$ subset. (5) Variants present in an unaffected member were excluded. (6) A sequence read depth filter retained variants whose read counts were $\geq 10$. (7) A pathogenicity filter retained variants scored as either possibly or probably damaging by Polyphen-2 (or unknown in the case of noncoding variants). Models 1-12 were built and named by Donald Primerano of Marshall University. Specific filters for each model are summarized in Table 2.

\section{Additional files}

Additional file 1. Variants present in selected mycobacterial susceptibility loci.

Additional file 2. Search for shared genes and associated disease states in this extended family.

\section{Abbreviations}

MAP: Mycobacterium avium ssp. paratuberculosis; UVBI: Ultraviolet blood irradiation using the $B$ and $C$ regions of the ultraviolet light spectrum; $C D$ : Crohn's disease; CRPS: complex regional pain syndrome; SNPs: single nucleotide polymphisms; SNVs: single nucleotide variants; IBD: inflammatory bowel disease; GWAS: genome wide association study; AD: autosomal dominant; AR: autosomal recessive; SA: splice acceptor; SD: splice donor.

\section{Acknowledgements}

The whole exome sequencing work and the data analysis were performed by Tempany Arbogast, Donald Primerano and Jun Fan of the Marshall University Genomics Core Facility. The Marshall University Genomics Core Facility is supported by the WV-INBRE Grant (P20GM103434), the COBRE ACCORD Grant (1P20GM121299) and the West Virginia Clinical and Translational Science Institute (WV-CTSI) Grant (2U54GM104942).

\section{Authors' contributions}

JTK conceived of the study and wrote the manuscript. JTK and MK obtained the consent of all participants and sought and obtained IRB approval for the study. MK and CW also helped designed the study and in the writing of the manuscript. All authors read and approved the final manuscript.

\section{Funding}

J. Todd Kuenstner paid for this work.

\section{Availability of data and materials}

Not applicable.

\section{Ethics approval and consent to participate}

This study conforms to the principles outlined in the Declaration of Helsinki. The goals of the study were explained to all of the participants and signed informed consent forms were obtained from all study subjects.

\section{Consent for publication}

All authors agree to the terms of the BioMed Central Copyright Manuscript and License Agreement and consent to the publication of this article.

\section{Competing interests}

Kuenstner is a shareholder of AVIcure Bioscience, a company with proprietary interests in Ultraviolet blood irradiation (UVBI) therapy. Kali and Welch have no competing interests.

\section{Author details}

1 Department of Pathology, Temple University Hospital, 3401 N. Broad St., Philadelphia, PA 19140, USA. ${ }^{2}$ CAMC Clinical Trials Center, 3100 MacCorkle Avenue S.E., Suite 806, Charleston, WV 25304, USA. ${ }^{3}$ Outcomes Research, 3100 MacCorkle Avenue S.E., Suite 806, Charleston, WV 25304, USA.

Received: 13 March 2019 Accepted: 30 May 2019

Published online: 20 June 2019

\section{References}

1. Naser SA, Ghobrial G, Romero C, Valentine JF. Culture of Mycobacterium avium subspecies paratuberculosis from the blood of patients with Crohn's disease. Lancet. 2004:364:1039-44.

2. Feller M, Huwiler K, Stephan R, Altpeter E, Shang A, Furrer H, Pfyffer GE, Jemmi T, Baumgartner A, Egger M. Mycobacterium avium subspecies paratuberculosis and Crohn's disease: a systematic review and metaanalysis. Lancet Infect Dis. 2007:7(9):607-13.

3. Abubakar I, Myhill D, Aliyu SH, Hunter PR. Detection of Mycobacterium avium subsp. paratuberculosis from patients with Crohn's disease using nucleic acid-based techniques: a systematic review and meta-analysis. Inflamm Bowel Dis. 2008;14(3):401-10.

4. Kuenstner JT, Naser S, Chamberlin W, Borody T, et al. The consensus from the Mycobacterium avium ssp. paratuberculosis (MAP) conference 2017. Front Public Health. 2017;5:208. https://doi.org/10.3389/fpubh .2017.00208.

5. Liu JZ, Anderson CA. Genetic studies of Crohn's disease: past, present and future. Best Pract Res Clin Gastroenterol. 2014;28(3):373-86. https://doi. org/10.1016/j.bpg.2014.04.009.

6. Pinedo PJ, Buergelt CD, Donovan GA, Melendez P, Morel L, Wu R, Langaee TY, Rae DO. Association between CARD15/NOD2 gene polymorphisms and paratuberculosis infection in cattle. Vet Microbiol. 2009;134(3-4):346-52.

7. Zhang FR, Huang W, Chen SM, Sun LD, Liu H, Li Y, et al. Genomewide association study of leprosy. N Engl J Med. 2009;361:2609-18. https://doi. org/10.1056/NEJMoa903753.

8. Schurr E, Gros P. A common genetic fingerprint in leprosy and Crohn's disease? N Engl J Med. 2009;361:2666-8. https://doi.org/10.1056/NEJMe 0910690

9. Jostins L, Ripke S, Weersma R, Duerr RH, McGovern DP, Hui KY, et al. Hostmicrobe interactions have shaped the genetic architecture of inflammatory bowel disease. Nature. 2012;491:119-24.

10. Kuenstner JT, Chamberlin W, Naser S, Collins MT, Dow CT, Aitken JM, Weg S, Telega G, John K, Haas D, Eckstein TM, Kali M, Welch C, Petrie T. Resolution of Crohn's disease and complex regional pain syndrome following treatment of paratuberculosis. World J Gastroenterol. 2015;21(13):404862. https://doi.org/10.3748/wjg.v21.i13.4048.

11. Ottenhoff THM, Dass RH, Yang N, Zhang MM, Wong HEE, et al. Genomewide expression profiling identifies type 1 interferon response pathways in active tuberculosis. PLoS ONE. 2012;7:e45839. https://doi.org/10.1371/ journal.pone.0045839.

12. Moeller M, Hoal E. Current findings, challenges and novel approaches in human genetic susceptibility to tuberculosis. Tuberculosis. 2010;90(2):7183. https://doi.org/10.1016/j.tube.2010.02.002.

13. Cobat $A$, et al. Two loci control tuberculin skin test reactivity in an area hyperendemic for tuberculosis. J Exp Med. 2009;206(12):2583-91.

14. Cobat $A$, et al. Tuberculin skin test negativity is under tight genetic control of chromosomal region 11p14-15 in settings with different tuberculosis endemic cities. J Infect Dis. 2015;211(2):317-21.

15. Belinky $F$, et al. Non-redundant compendium of human ncRNA genes in GeneCards. Bioinformatics. 2013;29(2):255-61.

16. Rappaport N, et al. MalaCards: a comprehensive automatically-mined database of human diseases. Curr Protocols Bioinf. 2014;47(1):1-24.

17. Lek $M$, et al. Analysis of protein-coding genetic variation in 60,706 humans. Nature. 2016;536:285. 
18. 1000 Genomes Project Consortium, Auton A, Brooks LD, Durbin RM, Garrison EP, Kang HM, Korbel JO, Marchini JL, McCarthy S, McVean $\mathrm{GA}$, et al. A global reference for human genetic variation. Nature. 2015;526(7571):68-74.

19. Schwarz JM, et al. MutationTaster evaluates disease-causing potential of sequence alterations. Nat Methods. 2010;7:575.

20. Adzhubei IA, et al. A method and server for predicting damaging missense mutations. Nat Methods. 2010;7(4):248-9.

21. Ng PC, Henikoff S. SIFT: predicting amino acid changes that affect protein function. Nucleic Acids Res. 2003;31(13):3812-4.

22. Molodecky NA, Soon IS, Rabi DM, Ghali WA, Ferris M, Chernoff G,

Benchimol El, Panaccione R, Ghosh S, Barkema HW, Kaplan GG. Increasing incidence and prevalence of the inflammatory bowel diseases with time, based on systematic review. Gastroenterology. 2012;142(1):46-54. https ://doi.org/10.1053/j.gastro.2011.10.001 (Epub 2011 Oct 14).
23. Johne's Disease on U.S. Dairies, 1991-2007. APHIS, United States Department of Agriculture. 2008.

24. Langmead B, Salzberg SL. Fast gapped-read alignment with Bowtie 2. Nat Methods. 2012;9(4):357-9.

25. Li H, Handsaker B, Wysoker A, Fennell T, Ruan J, Homer N, Marth G, Abecasis G, Durbin R, 1000 Genome Project Data Processing Subgroup. The Sequence alignment/map (SAM) format and SAMtools. Bioinformatics. 2009;25(16):2078-9. https://doi.org/10.1093/bioinformatics/btp352

(Epub 2009 Jun 8)

\section{Publisher's Note}

Springer Nature remains neutral with regard to jurisdictional claims in published maps and institutional affiliations.
Ready to submit your research? Choose BMC and benefit from:

- fast, convenient online submission

- thorough peer review by experienced researchers in your field

- rapid publication on acceptance

- support for research data, including large and complex data types

- gold Open Access which fosters wider collaboration and increased citations

- maximum visibility for your research: over 100M website views per year

At BMC, research is always in progress.

Learn more biomedcentral.com/submissions 